\title{
Límites estilométricos
}

en una miscelánea áurea: Favores de las musas de Sebastián Francisco de Medrano*

\section{Stylometric Limits in a Golden Age Miscellany: Favores de las musas by Sebastián Francisco de Medrano}

\section{Elena Martínez Carro}

https://orcid.org/0000-0001-6414-1724

Universidad Internacional de La Rioja (UNIR)

ESPAÑA

elena.martinez@unir.net

[Hipogrifo, (issn: 2328-1308), 9.1, 2021, pp. 159-174]

Recibido: 20-11-2020 / Aceptado: 22-12-2020

DOI: http://dx.doi.org/10.13035/H.2021.09.01.11

Resumen. La miscelánea Favores de las musas de Sebastián Francisco de Medrano contiene tres comedias diversas en cuanto a estilo, tratamiento y forma. La autoría de una de ellas, Lealtad, amistad y amor, ha sido ampliamente discutida por su coincidencia con la obra de Pérez de Montalbán, Amor, lealtad y amistad, también atribuida a Lope de Vega. A pesar de los numerosos estudios, el creador de la comedia ha seguido siendo una incógnita. Sin embargo, los análisis estilométricos comparativos entre las obras de la miscelánea han permitido una nueva aproximación al estudio filológico de esta comedia, así como a los límites de la investigación estilométrica respecto a las otras dos obras impresas en el volumen.

Palabras clave. Favores de las musas; Sebastián Francisco de Medrano; miscelánea áurea; Lealtad, amor y amistad; Venganzas de amor; El lucero eclipsado; estilometría.

Abstract. The miscellany Favores de las musas by Sebastián Francisco de Medrano contains three different plays in terms of style, treatment and form. The

* Este trabajo se inscribe en el marco del Grupo de Investigación de la UCM «Hibridismo literario y cultura áurea» (Ref. 970841). 
authorship of one of them, Lealtad, amistad y amor, has been widely discussed because it coincides with the work of Pérez de Montalbán, Amor, lealtad y amistad, also attributed to Lope de Vega. Despite of the numerous surveys, the creator of the comedy has remained an unknown. However, the comparative stylometric analyses between the works of the miscellany have allowed a new approach to the philological study of this play, as well as to the limits of stylometric research with respect to the other two works printed in the volume.

Keywords. Favores de las musas, Sebastián Francisco de Medrano; Golden Age miscellany; Lealtad, amor y amistad; Venganzas de amor; El lucero eclipsado; Stylometry.

\section{DEL AUTOR Y LA OBRA}

Favores de las musas, miscelánea de Sebastián Francisco de Medrano (15901653), fue editada en Milán en 1631 por Juan Bautista Malatesta como señala la descripción de su portada:

Favores de las mvsas / hechos a Don Sebastian / Francisco de Medrano. / En varias Rimas, y Comedias, que compuso en la mas / celebre Academia de Madrid donde fue / Presidente meritisimo. / Recopiladas por Don Alonso de Castillo / SoIorzano intimo amigo del Autor. Milan, luan Baptista Malatesta, a costa de Carlo Ferranti, 1631.

Recordaba La Barrera que el autor «las publicó en dos tomos, $8^{\circ}$, de los cuales el primero, que contiene cinco libros, repartidos por las cinco primeras musas, se imprimió antes y fue enviado a España y reimpreso en Madrid el propio año; y el segundo, estampado más tarde, quedó sepultado en el mar, habiendo naufragado el buque portador de la edición»' . No tenemos noticia de esta segunda parte, ni hemos localizado - hasta el momento- documentación que haga referencia al naufragio acontecido.

Las obras en verso en torno a las musas fueron compuestas para la Academia de Madrid y recogidas por Castillo Solórzano², gran amigo de Medrano y que entró a formar parte del grupo de poetas en 1619, dos años después de su fundación, como él mismo recordaba en sus versos:

Con lo cual a una academia que se fundó en Leganitos, me vine a entrar a poeta, si bien por lego novicio ${ }^{3}$. 
A pesar de que Sebastián Francisco de Medrano no fue uno de los poetas más insignes del momento, creó la llamada Academia de Medrano que muchos consideran coincidente con la Academia de Madrid y prestó su casa de Leganitos entre 1617 y 1622 para las reuniones. Formaron parte de esta Academia los nombres más ilustres del momento, como el mismo Medrano señalaba en la carta introductoria al tomo de los Favores de las musas, lista - por otra parte- incompleta de los participantes y en muchos coincidente con la Academia de Saldaña ${ }^{4}$. Entre ellos se encontraban Lope de Vega, Quevedo, Góngora, Tirso de Molina, Juan Ruiz de Alarcón, Luis Vélez de Guevara, Antonio Mira de Amescua, Juan Pérez de Montalbán, Calderón, Alonso de Castillo Solórzano y otros menos conocidos como Jerónimo de Villaizán, José Pellicer de Tovar y Gabriel Bocángel ${ }^{5}$.

Según comentaba el mismo Castillo Solórzano en la «Epístola a quien leyere» introductoria al volumen, Medrano era un adolescente cuando fundó la Academia. Había nacido «en Madrid, en el seno de una ilustre familia a finales del siglo Xvl; fue sacerdote y comisario de la Inquisición, actuando como censor oficial de comedias. Fue también capellán y tesorero del duque de Feria; desde 1622 perteneció a la congregación sacerdotal de San Pedro, que integraba a los curas naturales de Madrid, llegando a ser secretario y capellán mayor de esta institución»6.

Como la mayoría de los poetas del momento, participó en las justas en honor de la beatificación de San Isidro y fue premiado en el certamen poético de 1622 organizado por Lope de Vega en honor de San Ignacio de Loyola y San Francisco Javier?.

Tenemos noticia de su actividad como calificador por una censura localizada en la comedia manuscrita de Luis Belmonte Bermúdez, Casarse sin hablarse, que anteriormente fue aprobada por Juan Navarro de Espinosa y posteriormente corregida por él 8 .

Entre sus relaciones con los poetas de la corte destaca la amistad que al parecer tuvo con Lope de Vega. A pesar de que no fue un dramaturgo distinguido, su vida coincidió con el esplendor de la comedia nueva y los avatares de la revolución teatral, con la que no siempre estuvo de acuerdo. Sin embargo, su defensa de los preceptos clásicos no le impidieron la convivencia y amistad con el grande del momento. Como señalaba La Barrera, «tuvo muy íntimas relaciones con Lope de Vega, y la casualidad hizo que a su cuarto, situado en el seminario de los escoceses, fuese conducido aquel grande hombre cuando le acometió el desmayo, que precedió tres días a su muerte» ${ }^{9}$.

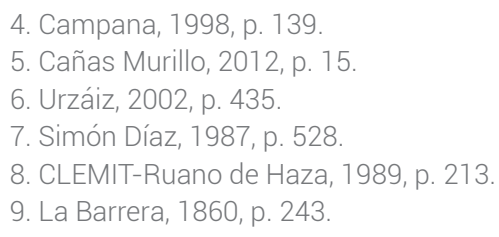


Lope de Vega se refirió a él elogiándolo en El Laurel de Apolo (1630) en la silva VII:

Don Sebastián Francisco de Medrano,

ilustre en nacimiento y en ingenio,

con diferente genio,

devoto deja el escribir profano ${ }^{10}$.

Medrano supo responderle en la Fama póstuma a la vida y muerte del doctor frey Lope Félix de Vega Carpio con un breve discurso evangélico y moral, basado en el capítulo 26 de Mateo, donde «el objetivo polémico eran los envidiosos» ${ }^{11}$. El mismo profundiza en esta exposición en la relación cercana que tenía con Lope: «Bien sabe el mundo cuánto lo amé y cómo le defendí, y así dirá el silencio lo que lloran los ojos [...]»12.

Aunque intervino en numerosos discursos panegíricos, donde la alabanza se veía tamizada por la reflexión evangélica en la que demostraba un gran conocimiento de la escritura bíblica por su formación religiosa, el epigrama dedicado a Lope ha destacado por su difusión:

Lope está, sepulcro, en vos,

porque escrito en vos se tope,

que si lo bueno es de Lope,

Lope, por bueno, es de Dios ${ }^{13}$.

La relación con Pérez de Montalbán también fue destacada y seguramente se había fraguado en la Academia Matritense y en las justas. El mismo Montalbán lo señalaba en el Para todos entre los ingenios elogiándolo por sus versos y por sus obras:

Don Sebastián Francisco de Medrano, de ingenio dulce, agudo y claro. Ha escrito con grande ostentación de su cordura muchos versos a varios asuntos y algunas comedias, no para que se representen, sino para que se sepa que las sabe hacer, y sobre todo dio a la imprenta unos soliloquios del Ave María, que juntamente con la devoción dan noticia de sus buenos estudios ${ }^{14}$.

Sin embargo, la amistad más avalada por las fuentes literarias que han llegado hasta nosotros es la que tuvo con Castillo Solórzano, quien recogió en el volumen de Favores de las musas sus poesías y obras teatrales. De hecho, fue posiblemente

Solórzano [quien] realiza su primer viaje a Italia en 1631 acompañando a su amigo don Francisco de Medrano, asistente del duque de Feria, para la publicación de Favores de las musas. No todos los críticos aceptan este primer viaje a Italia. Juliá Martínez cree que la intervención de Solórzano en la publicación de Favores de las musas se reduce a la conservación y reunión de las obras que Medrano

10. Fernández-Guerra y Orbe, 1871, p. 368.

11. Di Pastena, 2001, Introducción, p. LXXIV.

12. Pérez de Montalbán, Fama póstuma a la vida y muerte del doctor frey Lope Félix de Vega Carpio, p. 173.

13. Pérez de Montalbán, Fama póstuma a la vida y muerte del doctor frey Lope Félix de Vega Carpio, p. 173.

14. Pérez de Montalbán, Para todos, 1632, fol. 355v. 
leyó en la Academia de Madrid, de la que era su presidente, por lo que no fue necesario que viajara hasta Milán para la publicación de la obra. Pablo Jauralde Pou, en cambio, cree que Solórzano pudo efectivamente acompañar a su amigo a la capital lombarda, motivo que justificaría la ausencia del autor en el entierro de su señor, el marqués de Vélez, a finales de $1631^{15}$.

En cualquier caso, el prólogo de los Favores de las musas deja manifiesta la amistad mutua entre los dos poetas, tanto en la carta que Medrano dedica a Solórzano como por la respuesta de este en su «Epístola a quien leyere», con la intencionalidad de demostrar al mundo lo mismo que Montalbán había afirmado en su Para todos: «para que se sepa que las sabe hacer», afirmación que - por otro lado- resulta sospechosa por insistente.

Los comentarios sobre la originalidad de la obra no se hicieron esperar. El Príncipe de Esquilache dejó en uno de sus manuscritos unos versos que delatan la opinión que debía correr entre los círculos literarios:

Comentador de aquel Lucio Romano,

¿por qué de mi paciencia tanto abusas

si primero inventó cantar las musas

don Sebastián Francisco de Medrano?

$Y$ estando impreso en verso castellano

no tiene el robo manifiesto escusas

y más saliendo a luz la que rehúsas

año de treinta y uno, y en Milano.

¿Qué te hizo la Señora de Cetina

que dejas sepultado su marido

al pie de una inscripción grecolatina,

y con dos pedantismos atrevido

contra lo que el difunto determina

das al papel, lo que dejó al olvido? (fol. 101r) ${ }^{16}$

La referencia está hecha a la obra de Quevedo El parnaso español, monte en dos cumbres dividido, con las nueve musas castellanas donde se contienen poesías de Don Francisco de Quevedo Villegas, puesto que Medrano había establecido una estructura similar en su publicación.

\section{LAS OBRAS TEATRALES EN LOS FAVORES DE LAS MUSAS}

A pesar de que en la obra de Medrano destacan las poesías en torno a las cinco musas, son de especial interés para este estudio las comedias contenidas en el volumen, tanto por ser casi las únicas que se le conocen al autor, como por su discutida autoría en alguno de los casos. Solo son cuatro las obras teatrales cata-

15. Lepe García, 2017, p. 353

16. Mejía Sánchez y Ratto, 1953, pp. 360-361, comentaban a este respecto: «por los datos que trae deducimos que el soneto va enderezado contra José Antonio González de Salas, traductor de Las Troyanas de Séneca y editor de Quevedo (vs. 1 y 13-14). La obra de Medrano a que se alude, vs. 3-8, es la recopilación hecha por Castillo Solórzano: Favores de las musas». 
logadas de Sebastián Francisco de Medrano hasta el momento y tres de ellas se encuentran en los Favores de las musas: Las venganzas de amor, El lucero eclipsado, San Juan Bautista y Lealtad, amor y amistad. De la cuarta comedia solo se conoce una edición suelta de 1645, El nombre para la tierra y la vida para el cielo. Urzáiz también señala otra quinta comedia, dividida en dos partes, Los estragos por la hermosura, como atribuida «a Medrano en un Catálogo manuscrito de la BMPS, aunque este documento es poco fiable y en sus páginas se atribuye también a Corella Medrano» ${ }^{17}$. Finalmente, Vázquez Estévez especifica el diálogo el Triunfo de la alegría, también incluido en la miscelánea de los Favores como una comedia. Sin embargo, un acercamiento a las formas poéticas de los cinco Libros inspirados por las musas e incluidos en el volumen, impide ver este diálogo como una obra teatral al igual que las señaladas anteriormente.

Dada la escasa producción teatral de Medrano, las comedias de este volumen cobran relevancia por sus diferencias de estilo y formas entre sí, así como por su trayectoria bibliográfica y dudas autoriales. Todo ello hace de este volumen un ejemplar único para analizar los avances estilométricos y su alcance en las comedias de atribución dudosa, especialmente en misceláneas -como esta- donde se recogen obras diversas bajo una única autoría.

El lucero eclipsado, San Juan Bautista, tragedia sobre el martirio de San Juan Bautista, consta de cinco actos con dos cuadros escénicos cada uno de ellos, excepto el último acto con tres escenas. Cada acto finaliza con un coro que da uniformidad a la división de los cuadros, al tiempo que mantiene una estructura clásica frente a los planteamientos de la Nueva Comedia.

El tema del martirio, desarrollado escuetamente en los Evangelios de San Mateo y San $\operatorname{Marcos}^{18}$, ya había sido tratado con anterioridad en el drama litúrgico en latín y en los misterios de los siglos XV y XVI. La historia de una tragedia no era propicia para los nuevos tiempos de la comedia del siglo XVII. Sin embargo, Medrano, vio en el argumento la posibilidad de recordar los preceptos clásicos.

\section{Como comentaba Pérez Priego:}

Concebiría su tragedia como un puro ejercicio académico, dirigido más que nada a probar la viabilidad de los preceptos clasicistas, «para que se vea -como afirmaba Solórzano en el prólogo- que ay en España quien lo sabe hazer con todo primor»19.

Los personajes de esta obra son un reflejo de la historia bíblica con las licencias necesarias para la construcción teatral: La Verdad en hábito de ninfa; El rey Herodes, tetrarca de Galilea; Heliab, príncipe; Semei, Abner y Ananías, sus discípulos; Aser, tribuno; Gersón, mayordomo del rey Herodes; Herodías, reina; Hija de Herodías; San Juan, lucero del sol y el coro de inteligencias.

17. Urzáiz, 2002, p. 436.

18. Evangelio según San Mateo, 14, 1-12 y según San Marcos, 6, 14-29.

19. Pérez Priego, 1981, p. 187. 
El argumento de la comedia tiene similitudes con otras obras del momento, lo que llevó a Varey y Shergold ${ }^{20}$ a pensar que el manuscrito existente en la BNM, Ms. 15243, anónimo, con el título el Lucero del Sol, San Juan Bautista, podría tratarse de la obra de Medrano o de la Sirena del Jordán de Monroy.

La descripción por parte de Manos teatrales ${ }^{21}$ del manuscrito deja de nuevo patente las confusiones respecto a la comedia:

2) PM: «De mano del librero Matías Martínez, con algunas notas autógrafas del licenciado d. Francisco de Rojas. [...] Inédita, según Durán, que tiene referencias a la de Castro: "Degollación de San Juan Bautista". La Barrera dice que acaso sea de Medrano».

3) f. 1r: Rojas ha modificado el título en la portada, escrito originalmente «El luzero del Sol I Comedia nueua». Rojas ha añadido entre los dos renglones del título «San Juan Bautista» y «diuina»y ha entrado una «y» entre «Comedia»y «nueua» para que rece «Comedia diuina y nueua».

Urzáiz también señala la existencia de otro manuscrito Ms. 18074, fols. 184-231, de la BNM con el título La sirena del Jordán, San Juan Bautista y afirma que «en algunos catálogos se citan como dos comedias diferentes» pues «La Barrera cita el segundo título como auto sacramental»22. Con este mismo título también se conoce una impresa suelta publicada en 1679 y una representación en el corral del Príncipe en febrero de 1696, por la compañía de Vallejo ${ }^{23}$.

Esta comedia se encuentra en un tomo de manuscritos teatrales del siglo XVII encuadernados que Manos teatrales ${ }^{24}$ describe detalladamente:

1) PM: letra del s. XVII. En la primera hoja, de mano de Francisco de Rojas: «Señor San Juan Bautista. Diferente».

2) Copia limpia

E: hebreo - Que alegres fiestas son estas

A: La Sirena de el Jordan / de quien la comedia acaua».

Cabe señalar que ninguno de los dos manuscritos, atribuidos a Monroy, muestra similitudes aparentes con la obra de Medrano publicada en los Favores de las musas. Se trata de dos obras totalmente diferentes, aunque el personaje de San Juan Bautista en la obra de Medrano figure como 'lucero del sol', apreciación que analizó Pérez Priego en su estudio sobre la evolución de la tragedia del Bautista:

La comedia de Cristóbal de Monroy La Sirena del Jordán, en la que el dramaturgo sevillano trata de adaptar aquel tema trágico a los moldes de la comedia lopesca. Para ello no contaba con otra salida artística que la que le ofrecía el género

20. Varey y Shergold, 1989, p. 211.

21. Manos Teatrales se encuentra actualmente alojada en la página web https://www.manos.net/, consultada el 6 de octubre de 2020.

22. Urzáiz, 2002, p. 458.

23. Varey y Shergold, 1989, p. 211.

24. Manos teatrales, https://www.manos.net/, consultada el 6 de octubre de 2020. 
de las llamadas «comedias de cuerpo» inspiradas en las vidas de santos y de gran aceptación popular. Ateniéndose, pues, a las tres jornadas y recurriendo a los procedimientos tópicos del género («Pónense las niñeces del santo en primer lugar; luego, sus virtuosas acciones, y en la última jornada, sus milagros y muerte, con que la comedia viene a cobrar la perfección que entre ellos se requiere»). [...] Con tales características, la obra de Monroy resultaba una total mixtura de elementos que fundía episodios históricos y novelescos, sagrados y profanos; mezclaba reyes, santos, pastores y criados; y, ya mediante la adición de un conflicto amoroso, ya sobre todo mediante la reordenación artística de su final trágico, convertía en tragicómica la fábula grave y patética en que se había inspirado 25 .

A diferencia de esta mixtura, la obra de Medrano sigue la tragedia evangélica sin contemporizar con las nuevas modas del momento.

De las tres comedias que contiene la miscelánea, Las venganzas de amor es una de las más desconocidas en cuanto a origen y tradición bibliográfica. La comedia, dividida en tres actos, y sin escenas, es de carácter mitológico. Sus personajes dan muestra de ello: El dios Apolo; El dios Cupido; La diosa Diana; La diosa Venus; El dios Mercurio; El dios Marte; El dios Momo; Daphne, ninfa; Adonis, príncipe de Fenicia; Acteón, príncipe de Tebas; Músicos.

Más que una comedia al uso, se trata más bien de una declamación poética por parte cada uno de los dioses, en los que Medrano hace gala de la utilización del verso a la manera clásica y preceptista a través de sus diálogos, en una comedia que - por otra parte- se encuadra en una miscelánea de 'favores' hechos por las diversas musas: Calíope, Clío, Melpómene, Erato y Talía.

La comedia no parece tener problemas de autoría, aunque La Barrera señala el mismo título en singular, Venganza de amor, como de Don Francisco Pérez de Borja $^{26}$, del que apenas existen datos biográficos. Pudo ser natural de Salamanca y no se conoce su producción literaria. De hecho, solo figuran en el catálogo de La Barrera cuatro comedias de él, y de Venganza de amor no hay referencia, ni manuscrito, ni impreso localizado. La Barrera solo señalaba que se encontraba en la «librería de don Bartolomé José Gallardo un manuscrito original de una comedia suya, fechada en $1646 »^{27}$.

La comedia Lealtad, amor y amistad, de tipo palaciego, consta de tres actos sin escenas. Su trama gira en torno al amor que sienten dos galanes amigos hacia una dama de palacio. El protagonista se debate entre el amor que profesa a su amada y la lealtad que debe a su amigo. Sin apenas necesidad de escenografía, el conflicto se genera no solo por los sentimientos encontrados de los personajes, sino también por las confusiones producidas por la oscuridad y las cartas, tópicos comunes en este tipo de comedias a lo largo del siglo XVII. De las tres obras teatrales contenidas en los Favores, esta es la más estudiada y discutida en cuanto a su autoría debido al común título y argumento con la obra de Pérez de

25. Pérez Priego, 1981, pp. 189-190.

26. La Barrera, 1860, p. 590,

27. La Barrera, 1860, p. 301. 
Montalbán: Amor, lealtad y amistad, publicada en su Segundo tomo de comedias a cargo de su padre Alonso Pérez en el año 1638, un año después del fallecimiento del dramaturgo ${ }^{28}$.

La obra de Montalbán había sido anteriormente publicada en Zaragoza en 1632, un año después de la comedia de Medrano, en la Parte veinte y cinco de Diferentes Autores y en una reimpresión de la misma parte en 1633. Fue Dixon en 1961 quien primero señaló, en su célebre estudio sobre el Segundo tomo de comedias de Juan Pérez de Montalbán, su propósito de comprobar cuáles de estas comedias eran del dramaturgo ${ }^{29}$. Entre sus dudas se encontraba Amor, lealtad y amistad por sus grandes coincidencias con la obra de Medrano aunque Profeti no compartió esta opinión ${ }^{30}$.

La posibilidad de un hurto de la comedia planeaba constantemente sobre la interpretación de esta obra, pero creemos que un hecho así en vida de los dos autores habría ensombrecido la relación entre Medrano y Pérez de Montalbán. Ambos habían compartido acontecimientos y pertenecido a la Academia Matritense sin que esta publicación afectara aparentemente a su relación. De hecho, como ya hemos comentado, en el Para todos, Montalbán nombró entre los ingenios a Medrano elogiándolo por sus versos y por sus obras ${ }^{31}$.

A la incertidumbre sobre la autoría de la comedia, vino a sumarse la edición de Lealtad, amor y amistad a nombre de Lope de Vega en la Parte veinte y seis de Lope de Vega y otros, publicada en Zaragoza en 1645. Profeti32 recogió los datos del tomo por ser -hasta el momento- un volumen desaparecido que conoció La Barrera. Como afirmaba Germán Vega, «un volumen que bien podría haber sido un conglomerado de sueltas como en otros casos. Lo cual, además, explicaría los problemas de pervivencia que ha tenido» ${ }^{33}$.

Por todo ello, solo es posible la comparativa entre los textos de Medrano y de Montalbán. Del estudio entre ambas, Dixon se inclinó por la autoría de Medrano, tanto por su calidad y errores detectados en la obra de Montalbán, como por el tipo de versificación y respeto a las unidades clásicas que siempre fueron un reclamo para Medrano. La versificación compuesta por estrofas aliradas, la combinación de hexasílabos y heptasílabos y un porcentaje altísimo de redondillas, no son propias de Montalbán. A su vez la obra de Medrano es más perfecta en cuanto a la composición estrófica, por lo que parecería lógico pensar en esta obra como la primogénita del elenco. Sin embargo, desde ninguna de las comparativas filológicas realizadas hasta ahora se puede asegurar la autoría de ninguno de los dos autores.

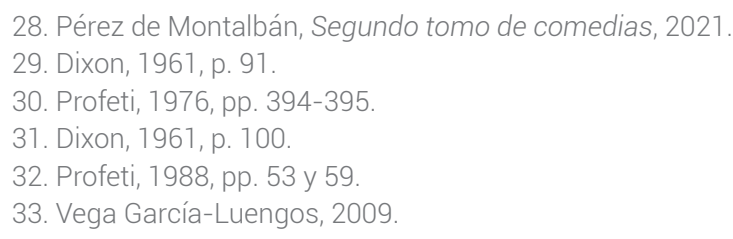


La repercusión de la obra por el tema fue emulada por autores como Castillo Solórzano, que en La traición en la amistad trató el argumento «como antítesis de la de su amigo Sebastián Francisco Medrano, Lealtad, amor y amistad»34, considerada como una de las tres mejores obras del autor ${ }^{35}$.

\section{ANÁLISIS ESTILOMÉTRICO DE LAS COMEDIAS DE LOS FAVORES DE LAS MUSAS}

La miscelánea en un solo volumen y editada a nombre de Sebastián Francisco de Medrano en 1631 como autor único de las tres comedias, época en la que vivían la mayor parte de los dramaturgos áureos a los que se podrían atribuir las obras y con los que convivía Medrano, sería suficiente argumento para afirmar que las tres obras son del autor por razones de lealtad y amistad hacia el grupo madrileño. Sin embargo, son tan numerosos los hurtos y plagios en la época - por razones de toda índole- que este argumento carece de fundamentación.

La variedad de estilos y formas de las comedias del volumen y especialmente los problemas de autoría de la comedia Lealtad, amor y amistad, tan tratados por Dixon ${ }^{36}$ y Profeti ${ }^{37}$ desde hace décadas, nos han llevado a estudiar los tres textos a la luz de la estilometría y sus últimos avances, como herramienta de apoyo filológico.

El estudio se ha realizado desde la base de datos ETSO (http://etso.es/) Estilometría aplicada al teatro del Siglo de Oro ${ }^{38}$, que permite la comparativa de las tres obras teatrales de los Favores de las musas con las 1292 comedias de la mayoría de los dramaturgos áureos recogidas en esta.

Como cabe recordar, la frecuencia de uso de palabras, o frecuencia léxica -que normalmente marcan el estilo de un autor - es detectada por sistemas y programas algorítmicos que buscan estas coincidencias ayudando a dilucidar la posible autoría de cada una de las obras. Cuanto mayor es la base comparativa, mayores son las posibilidades de determinar atribuciones dudosas como las que presentamos. Estudios recientes como los llevados a cabo por Germán Vega ${ }^{39}$, Álvaro Cuellar ${ }^{40} \mathrm{o}$ Claudia Demattè ${ }^{41}$ sobre la base de datos ETSO demuestran cómo la estilometría puede complementar los estudios filológicos y bibliográficos cuando estos plantean tradicionalmente dudas certeras sobre la autoría de determinadas comedias.

Aunque son muchos los programas algorítmicos que se han sucedido en los últimos años, inicialmente se trabaja en esta investigación con el paquete Stylo considerando las quinientas palabras más frecuentes de cada obra para mostrar

34. Navarro Durán, 2020, p. 218.

35. Antonio Matina hizo un juicio parco de las tres comedias contenidas en el volumen, señalando que «solamente la tercera es elegante». Ver Marcello, 2018, p. 316.

36. Dixon, 1961.

37. Profeti, 1976.

38. Cuéllar González y Vega García-Luengos, 2017

39. Vega García-Luengos, en prensa.

40. Cuéllar González, 2018 y en prensa.

41. Demattè, 2019 y en prensa. 
cuáles son las más cercanas a la comedia objeto de estudio42. Esto nos lleva a dilucidar entre los posibles autores marcados por las coincidencias léxicas de sus comedias. Solo después de examinar estas frecuencias podemos mostrar la cercanía entre las obras a través de gráficos de distancia como los siguientes, los cuales reflejan las obras del corpus más próximas léxicamente a la de nuestro interés.

Los resultados del primer análisis de coincidencias léxicas de las obras de Medrano muestran las siguientes gráficas:

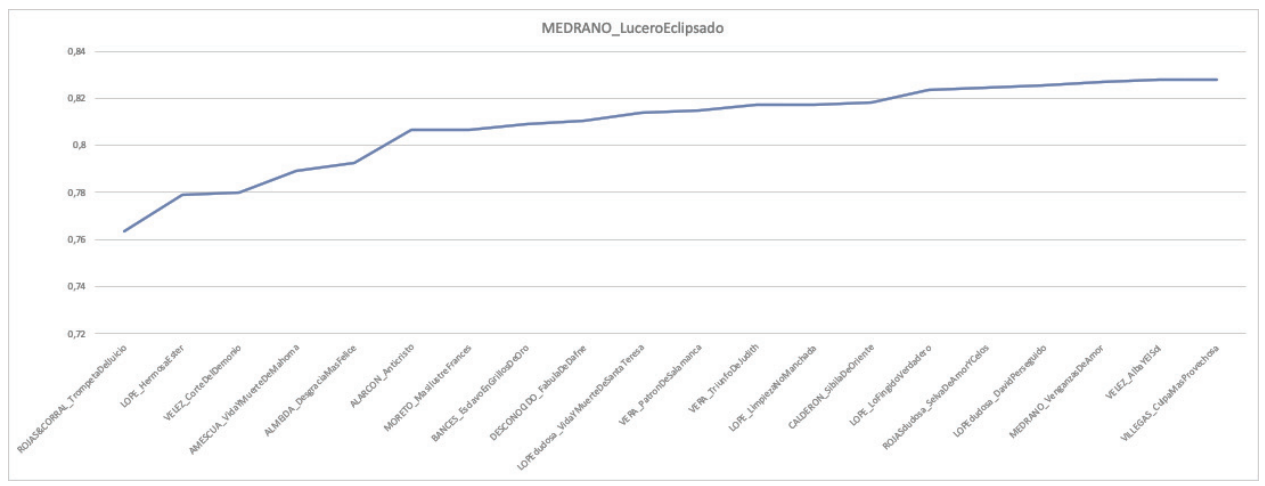

Fig. 1. Gráfico El lucero eclipsado

Obras más cercanas léxicamente a Lucero eclipsado

entre las 1292 obras del corpus ETSO a día 6 de octubre de 2020.

Stylo. Distancia Classic Delta. 500 MFW. 0\% culled

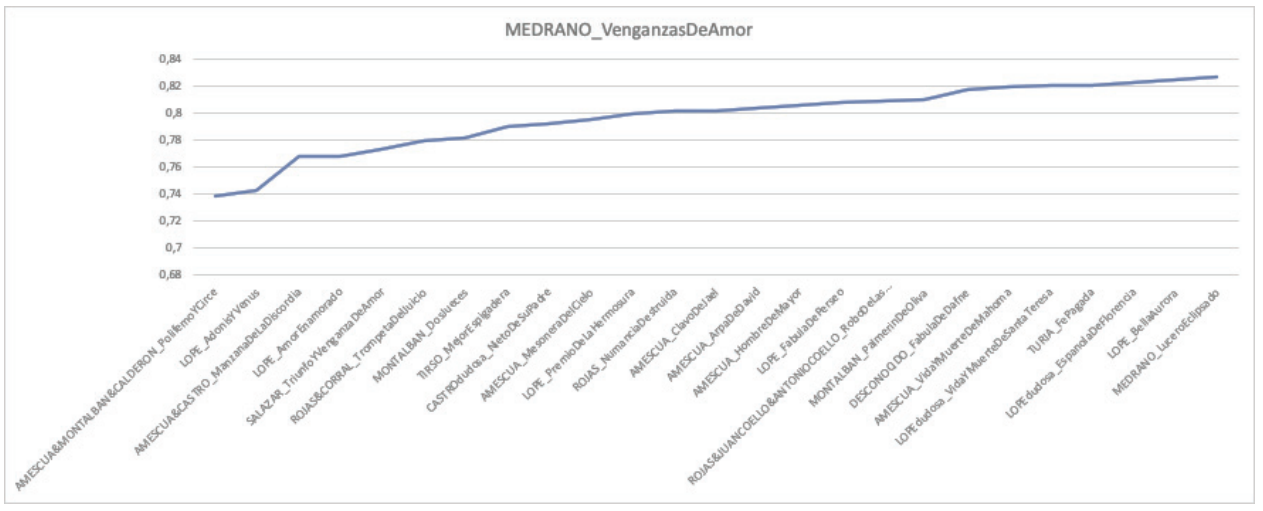

Fig. 2. Gráfico Venganzas de amor

Obras más cercanas léxicamente a Venganzas de amor

entre las 1292 obras del corpus ETSO a día 6 de octubre de 2020.

Stylo. Distancia Classic Delta. 500 MFW. 0\% culled

42. Para una mayor comprensión de los elementos cuantitativos seleccionados y su proceso en el paquete Stylo, ver Cuéllar González, en prensa; R CORE TEAM, 2020 Y EDER ET AL., 2016. 
Como puede observarse, las dos primeras comedias del volumen apenas tienen coincidencias léxicas entre sí como para ver similitudes entre ellas. El lucero eclipsado y Venganzas de amor muestran algunas semejanzas estilométricas, aunque tan alejadas entre sí que sería posible atribuirlas a un gran número de dramaturgos antes que a Medrano. Sin embargo, tampoco queda clara la autoría de ninguno de ellos por su diversidad y fluctuación en el estudio estilométrico. Desde esta perspectiva, el análisis solo aporta indefinición a la investigación de las dos obras y señala el estilo dispar de la mismas y la falta de rasgos comunes al autor. Cuando el estilo del dramaturgo es definido, las comedias tienden a agruparse como puede verse el gráfico 4, donde un gran número de obras aparecen atribuidas a Lope de Vega por el elevado número de coincidencias léxicas que tienen entre sí.

El análisis muestra que estas dos obras poseen frecuencias léxicas comunes con otros autores como Rojas Zorrilla, Mira de Amescua, Lope de Vega, Montalbán y Alarcón y que ambas entran en la concurrencia establecida, aunque sea remota. El hecho de que estas dos obras estén dentro de unos rangos de coincidencias léxicas podría acercar la autoría a Medrano, aunque con clarísimas influencias de los dramaturgos con los que compartió los años de la Academia Matritense, hasta el punto de emularlos.

Sin embargo, la tercera comedia, Lealtad, amor y amistad, no guarda ninguna conexión con las otras dos obras de Medrano como puede observarse en la figura 3 , donde ni siquiera aparecen de manera dispersa, como sucedía en los análisis anteriores, y sí tiene una total relación con el estilo de la obra de Montalbán, Amor, lealtad y amistad, donde la coincidencia que se aprecia es máxima.

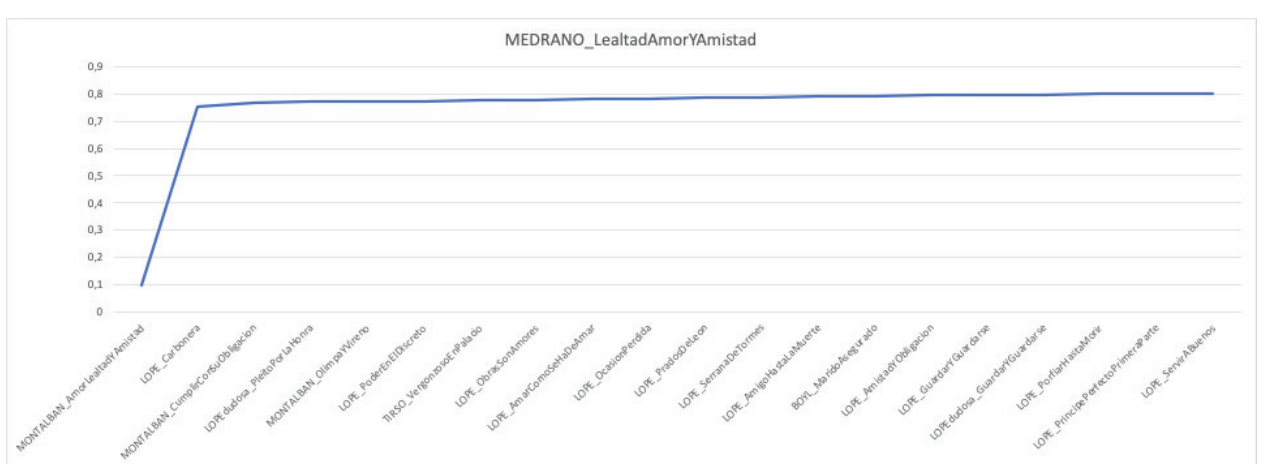

Fig. 3. Gráfico Lealtad, amor y amistad

Obras más cercanas léxicamente a Lealtad, amor y amistad entre las 1292 obras del corpus ETSO a día 6 de octubre de 2020.

Stylo. Distancia Classic Delta. 500 MFW. 0\% culled 


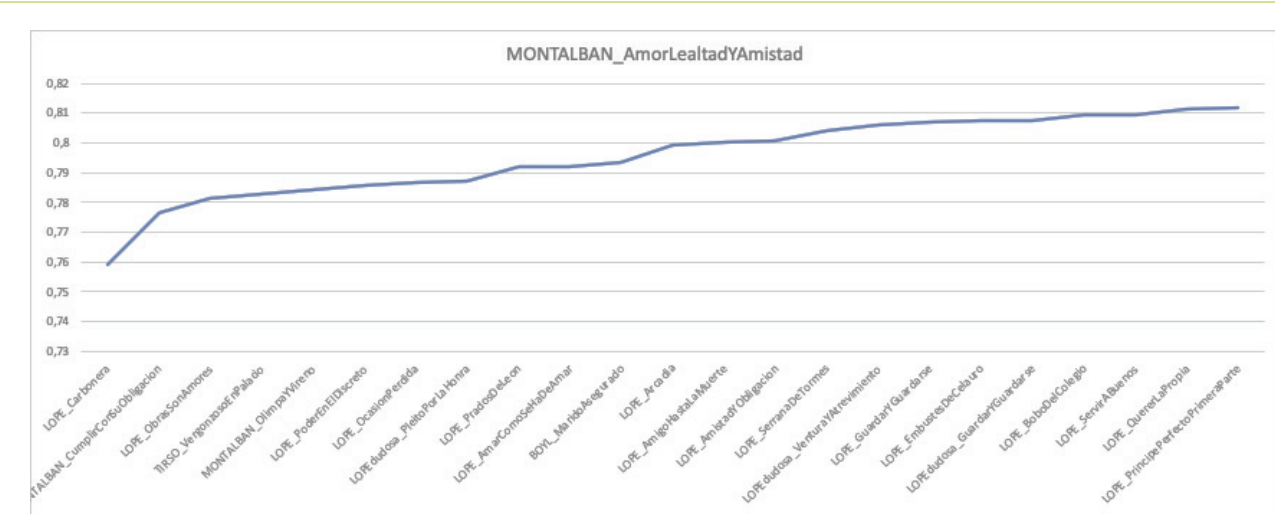

Fig. 4. Gráfico Amor, lealtad y amistad

Obras más cercanas léxicamente a Amor, lealtad y amistad entre las 1292 obras del corpus ETSO a día 6 de octubre de 2020. Stylo. Distancia Classic Delta. 500 MFW. 0\% culled

Si observamos el gráfico de la comedia Amor, lealtad y amistad (fig. 4), variante de la de Medrano pues son ambas coincidentes en un $90 \%$ de los versos, puede observarse que siguen sin figurar en la gráfica las otras dos obras del autor, mientras que esta comedia de atribución dudosa por su tradición bibliográfica sí se acerca a las obras de Montalbán, hasta el punto de situarse junto a dos obras de autoría segura del dramaturgo: Cumplir con su obligación y Olimpa y Vireno. Este dato parece esclarecedor para confirmar la autoría de Pérez de Montalbán sobre la comedia, como por otra parte vienen demostrando las investigaciones realizadas hasta ahora. El hecho de que no figuren ninguna de las otras obras de los Favores de las musas en el estudio estilométrico junto a Lealtad, amor y amistad, y sí lo haga junto a las de Montalbán, manifiesta la posible autoría de una de las comedias discutidas por Dixon y Profeti.

\section{CONCLUSIONES}

El análisis estilométrico de estas obras - aparentemente- puede defraudar a la pregunta inicial de investigación de este trabajo: ¿son las comedias contenidas en los Favores de las musas de Sebastián Francisco de Medrano? Efectivamente, el análisis no es concluyente y deja muchas incógnitas abiertas fruto de los límites actuales de los procesos estilométricos.

Sin embargo, no cabe duda de que la comparativa ha delimitado las incógnitas y abre nuevas perspectivas. Lejos de ser de ser decepcionantes los resultados, nos preguntamos si un análisis léxico entre las poesías contenidas en esta miscelánea y las obras teatrales nos aportarían más datos sobre la autoría de Venganzas de amor y El lucero eclipsado. Esta posibilidad está hasta el momento sin explorar puesto que la mezcla de dos géneros solo podría hacerse de manera independiente entre cada una de las comedias y las poesías de reconocida autoría. Es decir, un estudio así solo ayudaría a reafirmar una autoría y no a buscar una atribución dudosa. 
Por otro lado, programas actuales de estilometría trabajan sobre un número de obras acotadas como Principal Component Analysis (PCA), Cluster Analysis, Logistic Regression, etc., lo que hace difícil realizar una comparativa cuando el espectro de autores es tan amplio para un análisis fidedigno.

Quedan así pendientes de nuevas investigaciones estas dos comedias, seguros de que un análisis pormenorizado y comparativo con la poesía de Medrano nos abrirá otras perspectivas de análisis estilométrico.

Finalmente, el estudio parece haber apuntado hacia la atribución de la comedia Lealtad, amor y amistad como una autoría definida de Montalbán, editada por su padre en el Segundo tomo de comedias con el título Amor, lealtad y amistad, y anteriormente en Zaragoza en 1632, comedia que Medrano debió de conocer en los años de amistad con Montalbán y a la que seguramente añadió algunos versos propios. Desconocemos si este fue un ejercicio lúdico que realizaron entre ambos, o incluso en colaboración con Lope, por lo que Medrano la publicó un año antes que su amigo, pero el análisis estilométrico no muestra exactitudes con las otras dos comedias contenidas en el volumen y sí con la obra de Pérez de Montalbán, como - por otra parte- marcan las nuevas ediciones de la comedia.

Se manifiesta así, cómo el análisis estilométrico de las misceláneas áureas es un campo a explorar con el fin de examinar posibles concomitancias entre los diversos géneros cultivados por nuestros dramaturgos. La definición de atribuciones dudosas es uno de los ámbitos más importantes en el estudio del teatro del Siglo de Oro que necesariamente debe caminar hacia análisis avanzados semánticos que despejen dudas como las planteadas en este estudio.

\section{BiBLIOgRAFÍA}

Barrera y Leirado, Cayetano Alberto de la, Catálogo bibliográfico del teatro antiguo español, desde sus orígenes hasta mediados del siglo XVIII, Madrid, Rivadeneyra, 1860.

Campana, Patrizia, «Hacia una edición anotada de La Filomena de Lope de Vega: Epístola a Don Juan de Arguijo», en Edición y anotación de textos. Actas del Primer Congreso de Jóvenes Filólogos, A Coruña, Universidade da Coruña, 1998, pp. 135-144.

Cañas Murillo, Jesús, "Corte y academias literarias en la España de Felipe IV》, Anuario de Estudios Filológicos, 35, 2012, pp. 5-26.

CLEMIT, Urzáiz Tortajada, Héctor et al., Censuras y licencias en manuscritos e impresos teatrales. CLEMIT. Publicación en web: http://buscador.clemit.es.

Cuéllar González, Álvaro, «La necesidad de la validación cruzada en Stylo y cómo programar en R», Caracteres. Estudios culturales y críticos de la esfera digital, 7.2, 2018, pp. 301-320. 
Cuéllar González, Álvaro, «Stylometry and Spanish Golden Age Theatre: An Evaluation of Authorship Attribution in a Control Group of Undisputed Plays», en Digital Stylistics in Romance Studies and Beyond, Heidelberg, Heidelberg University Press, en prensa.

Cuéllar González, Álvaro, y Germán Vega García-Luengos, ETSO: Estilometría aplicada al Teatro del Siglo de Oro, 2017. Visitada el 30 de septiembre de 2020.

Demattè, Claudia, «Una nueva comedia en colaboración entre ¿Calderón?, Rojas Zorrilla y Montalbán: Empezar a ser amigos a la luz del análisis estilométrico», Rilce. Revista de filología hispánica, 35.3, 2019, pp. 852-874.

Demattè, Claudia, «El Segundo tomo de comedias de Juan Pérez de Montalbán: hacia un mapa estilométrico de las comedias auténticas, dudosas y ajenas», Talía, en prensa.

Di Pastena, Enrico, edición crítica, estudio y notas de Juan Pérez de Montalbán, Fama póstuma a la vida y muerte del doctor frey Lope Félix de Vega Carpio y elogios panegíricos a la inmortalidad de su nombre, Pisa, Edizioni ETS, 2001.

Dixon, Víctor, «Juan Pérez de Montalbán's Segundo tomo de las comedias», Hispanic Review, 29, 1961, pp. 91-109.

Eder, Maciej, Rybicki, Jan, y Kestemont, Mike, «Stylometry with R: a Package for Computational Text Analysis», R Journal, 8.1, 2016, pp. 107-121, https:// journal.r-project.org/archive/2016/RJ-2016-007/index.html.

Fernández-Guerra y Orbe, Luis, Don Juan Ruiz de Alarcón y Mendoza, Madrid, Rivadeneyra, 1871.

Lepe García, M. ${ }^{a}$ Rocío, «El último Castillo Solórzano: hacia un modelo innovador del marco narrativo», en Compostella Aurea. Actas del VIII Congreso de la AISO, ed. Antonio Azaústre y Santiago Fernández Mosquera, Santiago de Compostela, Universidade de Santiago de Compostela, 2011, pp. 347-354.

Manos teatrales, Greer, Margaret R. et al., Manos teatrales. Publicación en web: https://www.manos.net/.

Marcello, Elena E., «Lecturas españolas en la Nápoles virreinal: el "Myrobiblon..." de Antonio Matina», Studia Aurea, 12, 2018, pp. 307-343.

Mejía Sánchez, Ernesto, y Ratto, Luis Alberto, «Poesías inéditas del Príncipe de Esquilache», Nueva Revista de Filología Hispánica, 7, 1953, pp. 352-363.

Navarro Durán, Rosa, «La traición en la amistad de María de Zayas: un juego literario de Castillo Solórzano», Anagnórisis, 21, 2020, pp. 190-221.

Pérez de Montalbán, Juan, Fama póstuma a la vida y muerte del doctor frey Lope Félix de Vega Carpio y elogios panegíricos a la inmortalidad de su nombre, ed. Enrico Di Pastena, Pisa, Edizioni ETS, 2001.

Pérez de Montalbán, Juan, Para todos, a costa de Alonso Pérez, Madrid, Imprenta del Reino, 1632. 
Pérez de Montalbán, Juan, Segundo tomo de comedias, ed. Claudia Demattè, vol. 2.3: Amor, lealtad y amistad, ed. Elena Martínez Carro, Kassel, Reichenberger, 2021, pp. 269-406.

Pérez Priego, Miguel Ángel, «La cabeza de Juan el Bautista, una tradición teatral», Anuario de Estudios Filológicos, 4, 1981, pp. 183-195.

Profeti, Maria Grazia, Per una bibliografia di Juan Pérez de Montalbán, Verona, Università di Padova, 1976.

Profeti, Maria Grazia, La collezione «Diferentes Autores», Kassel, Edition Reichenberger, 1988.

R Core Team, R: A Language and Environment for Statistical Computing, R Foundation for Statistical Computing, 2020, https://www.R-project.org.

Rodríguez Mansilla, Fernando, «El romance a Don Juan de Espina de Castillo Solórzano», Calíope, 14.2, 2008, pp. 5-26.

Ruano de la Haza, José María, «Dos censores de comedias de mediados del siglo XVII», en Estudios sobre Calderón y el teatro de la Edad de Oro. Homenaje a Kurt y Roswitha Reichenberger, ed. Francisco Mundi Pedret et al., Barcelona, PPU, 1989, pp. 201-229.

Simón Díaz, José, «Fiesta y literatura en el Colegio Imperial de Madrid», Dicenda, 6, 1987, pp. 525-537.

Urzáiz, Héctor, Catálogo de autores teatrales del siglo XVII, Madrid, FUE, 2002.

Varey, John E., y Shergold, Norman D., Comedias en Madrid, 1603-1709: Repertorio y estudio bibliográfico, London, Tamesis, 1989.

Vega García-Luengos, Germán, «Enredar con Lope: indagaciones a partir de la comedia de Dos agravios sin ofensa», Alicante, Biblioteca Virtual Cervantes, 2009, http://www.cervantesvirtual.com/obra-visor/enredar-con-lope-indagaciones-a-partir-de-la-comedia-de-dos-agravios-sin-ofensa-0/html/0216e58c82b2-11df-acc7-002185ce6064_5.html\#I_0_.

Vega García-Luengos, Germán, «Juan Ruiz de Alarcón recupera La monja alférez», en Sor Juana Inés de la Cruz y el teatro novohispano. XLII Jornadas de teatro clásico de Almagro. Almagro, 9 al 11 de julio de 2019, ed. Rafael González Cañal y Elena E. Marcello, Ciudad Real, Ediciones de la Universidad de Castilla-La Mancha, en prensa. 